\title{
Decision Support System for the Diagnosis of Parkinson's Disease
}

\author{
Anders Ericsson, Markus Nowak Lonsdale, Kalle Astrom, \\ Lars Edenbrandt, and Lars Friberg \\ No Institute Given
}

\begin{abstract}
Recently new nuclear medical diagnostic imaging methods have been introduced by which it is possible to diagnose Parkinson's disease, PD, at an early stage. The binding of a specific receptor ligand [123I]-FP-CIT in specific structures of the human brain is visualized by way of single photon emission computerized tomography, SPECT. The interpretation of the SPECT data can be accessed by visual analysis and manual quantification methods. We have developed a computer aided automatic decision support system in attempt to facilitate the diagnosis of Parkinson's disease from the acquired SPECT images. A rigid model was used for the segmentation of the basal ganglia of the human brain. The aim of the study was to develop an automated method for quantification and classification of the images.

The study comprises SPECT scans 89 patients, who underwent a [123I]-FP-CIT examination because of suspected Parkinson's disease. An experienced nuclear medicine physician interpreted the images and diagnosed 65 of the patients as most likely suffering from Parkinson's disease.

The method included the following steps; (i) segmentation of basal ganglia by fitting a rigid 3D model to the image, (ii) extraction of 17 features based on image intensity distribution in the basal ganglia and a reference based on image intensity distribution outside the basal ganglia, (iii) classification using Support Vector Machine (SVM).

The classification based on the automated method showed a true acceptance of $96.9 \%$ and a true rejection of $91.6 \%$. The classification based on a manual quantification method gave a true acceptance of $98.5 \%$ and a true rejection of $100 \%$. The method proposed here is fully automatic and it makes use of the full 3D data set in contrast to a method that is widely used at hospitals today which only uses a few 2D image slices.
\end{abstract}

\section{Introduction}

Parkinson's disease (PD) is a neurological movement disorder associated with slow movements, tremor, rigidity and postural instability. The disease can occur before the age of 40 , but more commonly it affects people in their $60 \mathrm{~s}$. The first symptoms can be very delicate, e.g., slight tremor of one hand. The disease usually progress slowly over years but in the late stages PD patients may become severely affected and wheelchair bound. There is no cure for PD, but patients may benefit from anti-parkinsonian medication. 
An early and correct diagnosis of PD is important for the management of patients. Until recently the diagnosis solely was based on clinical assessment and clinical rating scales. The initial diagnoses of PD made by general practitioners have shown to be incorrect in $24 \%$ to $35 \%$ of the cases [1]. A reliable diagnostic test, which could be used to differentiate between different tremor disorders, would therefore be of great value. PD is caused by degeneration of a part of the brain called substantia nigra. The dopamine producing neurons projects to a nearby structure called the basal ganglia where dopamine is released. The cell membrane of these neurons contain dopamine re-uptake receptor sites (dopamine transporters, DAT). The number of the dopamine producing neurons decrease and the reduction of receptors can be assessed with [123I]-FP-CIT, which is a recently introduced diagnostic method [2] [3] . The radiotracer is injected intravenously transported with the blood to the brain where it binds to the presynaptic dopamine re-uptake site receptors. After 3 hours when an equilibrium between free and bound receptor ligand is reached 3-dimensional images of the brain are obtained using a gamma camera. The interpretation of the examination is based on both a visual assessment and a quantification of the images in order to assist the physician in the interpretation process. The binding of radiotracers in the basal ganglia and other brain areas can be assessed by manual positioning of regions of interest (ROI). The operator selects from the image a few slices with the highest uptake and the slices are added. Thereafter a set of predefined ROIs is superimposed over the basal ganglia bilaterally and a reference region - usually the visual cortex in the posterior aspect of the brain. The average number of counts of the ROIs are used as an indicator of the number of receptors in different parts of the brain. This manual quantification method has proven effective in more that a 1000 [123I]-FP-CIT images that it has been applied to. However, it is time consuming and operator dependent. In addition only a portion of the 3 -dimensional volume is considered in the calculations.

The aim of the present study was twofold. First to develop an automated method for quantification and classification of [123I]-FP-CIT images. The method should be based on a 3-dimensional analysis. Second to compare the performance of the new method with that of a manual quantification method.

\section{Material and Method}

\subsection{Material}

The material have been [123I]-FP-CIT-images obtained at investigations at the Department of Clinical Physiology and Nuclear Medicine at Bispebjerg Hospital. In the database there are images from 89 patients that went through a diagnostic investigation because of suspected PD. The patients had a mean age of 68 years (range 21 - 82). The final diagnosis based on the interpretation by an experienced nuclear medicine physician was PD in 65 cases and non PD in 24 cases. 


\subsection{Method}

A dose of $185-225 \mathrm{MBq}$ of [123I]-FP-CIT was injected intravenously and after three hours data acquisition was performed with a three-headed gamma camera (Prism XP3000, Maconi/Philips). Emission data were acquired with a full 360 degree rotation with simultaneously acquisition of transmission data from a $159 \mathrm{Gd}$-source. Image processing was performed using iterative reconstruction with scatter and non-uniform attenuation correction. The iterative algorithm (ordered subsets) OS-EM was used. The number of iterations was 20 for the transmission scans and 4 for the emission data. The chosen number of iterations was based on pilot studies. Resolution in trans-axial plane was $6 \mathrm{~mm}$ of the reconstructed slices.

The automated method for quantification and classification of the brain images consists of three main steps: segmentation of the basal ganglia, feature extraction from these regions, and classification of the images. The aim with this work has been to automatically identify images with abnormal receptor radio ligand distribution indicating presences of idiopathic Parkinson's disease or related Parkinson Plus syndromes. In the analysis two main problems was considered: 1) extracting automatically the outline of the basal ganglia and its substructures (caudate nucleus and putamen) and 2) training a classifier on those features.

Algorithm for automatic classification of Parkinson's disease.

\section{SEGMENTATION}

1. Initiate the model

The first step is to initiate the model of the basal ganglia.

2. Fit the model to data

The model is fitted to data by running an optimisation scheme.

\section{QUANTIFICATION}

3. Measure the intensity distribution

Once the model is fitted, the mean intensity is measured inside four of the regions: left and right putamen and left and right caudatus.

4. Normalisation

To be able to normalise; obtain a reference value by taking the median of the intensity outside the basal ganglia.

5. Retrieve features

Retrieve features from measures.

\section{CLASSIFICATION}

6. Classify using SVM

Classify the features using Support Vector Machines. 
Above is a short overview of the method. The main algorithmic development has been done for automatic segmentation of the basal ganglia and automatic feature extraction from those regions. These features are used for the training and classification procedures.

\section{$2.3 \quad$ Segmentation}

The first step is to segment out the basal ganglia. This is done by optimising the fit of a 3D-model of the basal ganglia.

(1) Initiate the Model. Before optimising the model parameters (position and rotation), an initial location of the model must be found. Since the intensity is high in the basal ganglia compared to other brain structure, the centre of mass of the brain corresponds very well to the location of the basal ganglia.

The centre of mass is calculated in the following way: Let $\mathcal{I}$ be the three dimensional image divided into voxels. Let $\mathcal{J}$ be the set of indices corresponding to the $M$ voxels with highest intensity. $M$ is chosen so that around $20 \%$ of the voxels are considered. Then the centre of mass

$\mathbf{C}_{m}=\left[x_{m}, y_{m}, z_{m}\right]$ is retrieved by

$$
x_{m}=\frac{\sum_{i \in \mathcal{J}} \mathcal{I}(i) x(i)}{\sum_{i \in \mathcal{J}} \mathcal{I}(i)}, y_{m}=\frac{\sum_{i \in \mathcal{J}} \mathcal{I}(i) y(i)}{\sum_{i \in \mathcal{J}} \mathcal{I}(i)}, z_{m}=\frac{\sum_{i \in \mathcal{J}} \mathcal{I}(i) z(i)}{\sum_{i \in \mathcal{J}} \mathcal{I}(i)},
$$

where $x(i)$ is the $\mathrm{x}$-coordinate of the voxel with index $i$.

(2) Fit the Model to Data. First a brief explanation of the model and then the optimisation procedure to fit the model to the image.

The Model. The model, which consists of two volumes of 3D-voxles, see Figure 1 , is rigid, i.e. has no shape parameters. It has eight degrees of freedom. Except for translation and rotation (six degrees) there are two other parameters, $\alpha$ and $\delta$. The distance between the left and the right side is $\delta$, and the skewness angle between the left and the right side is $\alpha$. The model consists of four regions: left putamen, right putamen, left caudatus and right caudatus, see Figure 1. Each region was defined by a number of 3D-points. These 3D-points were defined from the basal ganglia segmented manually from a 'T1-Single- Subject MR' template distributed with software package SPM'99 (http://www.fil.ion.ucl.ac.uk/spm).

Let $\mathcal{M}_{l}$ be the set containing the points of the left hand side of the model (left striatum) and let $\mathcal{M}_{r}$ be the corresponding right hand side. The operator $\mathbf{R}_{\alpha}$ rotates points in a set $\alpha$ radians clockwise in the z-plane. The operator $\mathbf{R}_{\theta, \varphi, \psi}$ rotates points in a set and $\mathbf{t}$ and $\mathbf{t}_{\delta}$ are translation vectors. Then the model $\mathcal{M}(\mathbf{t}, \mathbf{R}, \alpha, \delta)$ is stated

$$
\mathcal{M}=\mathbf{R}\left(\mathbf{R}_{\alpha / 2} \mathcal{M}_{l}-\mathbf{t}_{\delta / 2} \cup \mathbf{R}_{-\alpha / 2} \mathcal{M}_{r}+\mathbf{t}_{\delta / 2}\right)+\mathbf{t} .
$$



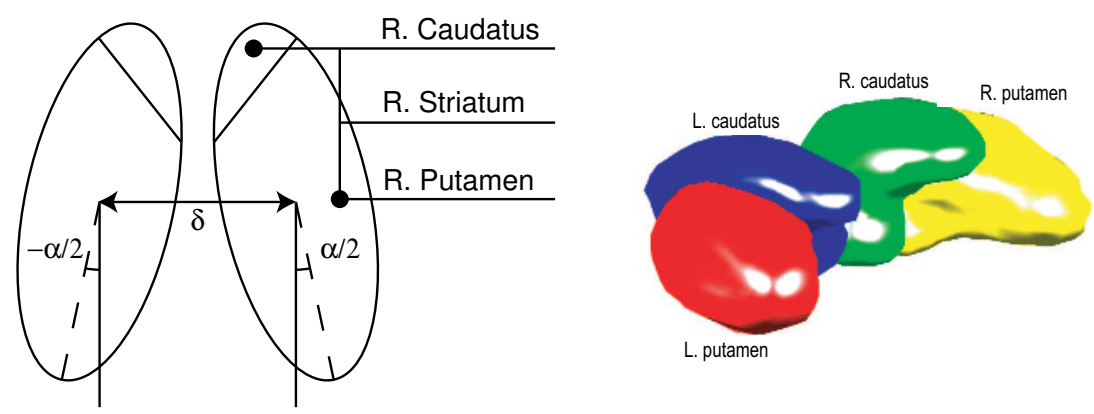

Fig. 1. To the left a schematic figure of the model. To the right a 3D-image of the model, where the $3 \mathrm{D}$ points are defined from an MR-image

Optimisation Scheme. The fitting of the model to data is stated as an optimization problem. Depending on the properties of the isotope (DAT), the intensity is highest at the location of the basal ganglia. The model is therefore fitted to the data by maximizing the intensity contained inside the two volumes. This can be expressed as a constrained-linear maximization problem. The 3D voxel data contains also structures in the lower part of the head below the brain. In severe cases where the intensity of the basal ganglia is relatively low it is important to constrain the search from the lower parts of the data. In some severe cases with very low radioisotope uptake in the basal ganglia the non-specific uptake in the parotid glands becomes very prominent showing a relatively high intensity.

The goal function is chosen to be the integrated intensity inside the two volumes. Let $\mathcal{M}$ be the model in (1) and $\mathcal{I}$ is the $3 \mathrm{D}$ voxel image, then the goal function $f$ is defined

$$
f(\mathbf{t}, \mathbf{R}, \alpha, \delta)=\sum_{x \in \mathcal{M}(\mathbf{t}, \mathbf{R}, \alpha, \delta):} \mathcal{I}(x) .
$$

The optimization problem becomes

$$
\max _{(\mathbf{t}, \mathbf{R}, \alpha, \delta)} f(\mathbf{t}, \mathbf{R}, \alpha, \delta)
$$

where

$$
\mathbf{t} \geq \mathbf{t}_{\min }, \mathbf{t} \leq \mathbf{t}_{\max }, \alpha \geq 0, \alpha \leq \alpha_{\max }, \delta \geq \delta_{\min }, \delta \leq \delta_{\max } .
$$

Several different optimization methods could be considered, for example constrained Levenberg-Marquardt. Here steepest descent is used and it turns out to work well. The result is that the model fits to the nearest local maxima from the initialization point. For most cases this is a global maximum. For severe cases of Parkinson the initialization point is important. The optimization might fail for severe cases, but they are easy to interpret, for example by looking at the maximum intensity relative to the mean intensity. 


\subsection{Quantification}

(3) Measure the Mean Intensity. Once the model has been fitted to data, the mean intensity inside the four regions defined by the model is measured. The sets representing left and right putamina and left and right caudate nuclei are $\mathcal{M}_{p_{l}}, \mathcal{M}_{p_{r}}, \mathcal{M}_{c_{l}}$ and $\mathcal{M}_{c_{r}}$. The mean intensity inside the left putamen $p_{l}$ is retrieved as

$$
p_{l}=\frac{\sum_{x \in \mathcal{M}} \mathcal{I}(x)}{\sum_{x \in \mathcal{M}} 1} .
$$

The other regions are treated analogously.

(4) Normalisation. The radiotracer binds to the dopamine transporters (reuptake sites) in the basal ganglia. In addition to this specific binding there is also a non-specific binding in the rest of the brain. In order to determine the specific binding the data for the selected volumes of interest, VOI, should be corrected for non-specific binding. The median count rate outside the basal ganglia, i.e. the rest of the brain, was used as the reference value. This value was used to calculate the specific-to-nonspecific binding ratio.

(5) Retrieve Features from Measures. The features that are used are described in Table 1 . The mean intensity per voxel for left and right caudate and for left and right putamen is denoted $c_{l}, c_{r}, p_{l}$ and $p_{r}$ respectively. The reference value is $m$.

Table 1. Table that explains all features being used

\begin{tabular}{|l|l|c|}
\hline Feature & Expression & Explanation \\
\hline$x_{1}$ & $c_{l}-m$ & Subtract median radiation \\
$x_{2}$ & $c_{r}-m$ & $-"-$ \\
$x_{3}$ & $p_{l}-m$ & $-"-$ \\
$x_{4}$ & $p_{r}-m$ & $-"-$ \\
$x_{5}$ & $m$ & Median radiation \\
$x_{6}$ & $x_{1} / m$ & Normalisation \\
$x_{7}$ & $x_{2} / m$ & $-"-$ \\
$x_{8}$ & $x_{3} / m$ & $-"-$ \\
$x_{9}$ & $x_{4} / m$ & $-"-$ \\
$x_{10}$ & $p_{r} / c_{r}$ & Quotient between right putamen and caudatus \\
$x_{11}$ & $p_{l} / c_{l}$ & Quotient between left putamen and caudatus \\
$x_{12}$ & $\frac{c_{l}+p_{l}-c_{r}+p_{r}}{c_{l}+p_{l}}$ & Quotient between left and right striatum \\
$x_{13}$ & $\left(x_{1}-x_{2}\right) / x_{1}$ & Quotient between left and right caudatus \\
$x_{14}$ & $\left(x_{3}-x_{4}\right) / x_{3}$ & Quotient between left and right putamen \\
$x_{15}$ & \multicolumn{2}{|l}{ Age } \\
$x_{16}$ & \multicolumn{2}{|l}{ Gender } \\
\hline
\end{tabular}




\subsection{Classification}

(6) Classification Using SVM. A Support Vector Classifier was chosen to discriminate the groups abnormal images likely to reflect parkinsonism and images from patients less likely to suffer from parkinsonism. SVC is advantageous to use when the underlying distribution is unknown. Ma, Zhao, and Ahalt's Implementation of Support Vector Machines has been used (the OSU-SVM toolbox for MATLAB [4]).

\subsection{Performance Measure}

The Support Vector Machines give for each sample a decision value. The samples are classified to one class or the other depending on the decision value (if it is below or above the threshold). By varying the threshold a receiver operating characteristic (ROC) curve was obtained. The performance of the classification was measured as the area under the ROC curve.

\section{$3 \quad$ Results}

The proposed algorithm has been tested on 89 cases consisting of 65 investigations showing abnormal [123I]-FP-CIT distribution (likely parkinsonism) and 24 patients with normal normal [123I]- FP-CIT distribution (unlikely parkinsonism). Using the features automatically extracted from these subjects a Support Vector Network has been trained. In Table 2 the results from leave-one-out tests on these 89 cases are given. Also the results from doing the same type of classification using the features of the manual method are presented. Specificity and sensitivity are two terms commonly used in medicine and are other words for true rejection rate and true acceptance rate, respectively.

Table 2. The results of leave-one-out tests using different models and under different filtering

\begin{tabular}{|r|r|r|r|}
\hline Method & Rate & Specificity & Sensitivity \\
\hline Automatic method & $95.5 \%$ & $91.7 \%$ & $96.9 \%$ \\
Semi automatic method & $98.9 \%$ & $100 \%$ & $98.5 \%$ \\
\hline
\end{tabular}

The performance was also measured from the ROC-curves, where the area under the graph is a measure of performance. In the novel method the area was 0.97 and in the semi-automatic system the area was 0.94 , see Figure 2.

To be able to visualize the separation between the two groups, the feature space has been projected onto a two dimensional plane. This is done by selecting the two main axes in the high dimensional feature space. Singular value decomposition (SVD) can be used. If $F$ is the feature matrix, each column corresponds to one patient. SVD gives, $F=U S V^{T}$, where $S V^{T}$ is the new coordinates in 

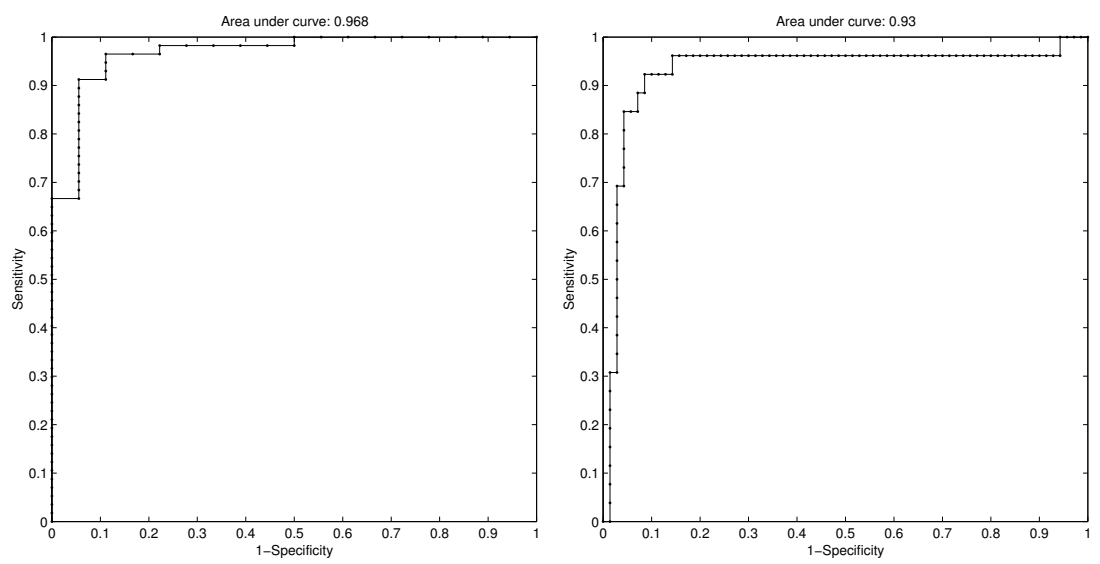

Fig. 2. The ROC-curve for Algorithm 3 (left) and the ROC-curve for the semiautomatic method (right)

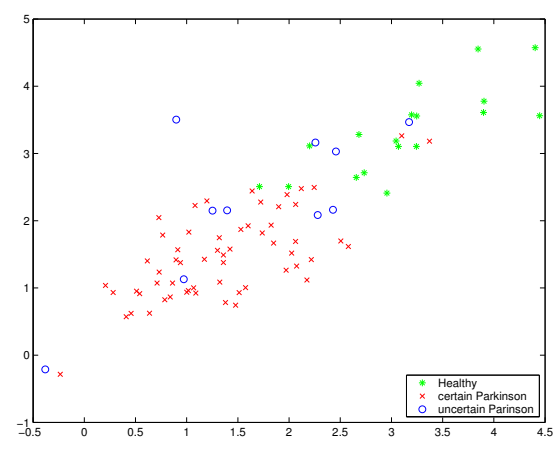

Fig. 3. Even when the feature space has been projected into a plane of two dimensions the separation of the groups Parkinson/non Parkinson is visible

the base $U$. The first and the second column vector of $U$ corresponds to the highest and the second highest singular value. Projecting the feature space $F$ onto the space spanned by $V$ these two vectors gives the best representation in two dimensions. The coordinates corresponding two these vectors are the two first rows in $S V^{T}$. Even in two dimensions the separation of the groups Parkinson/non Parkinson is visible, see Figure 3.

\section{Discussion}

\subsection{Segmentation}

The segmentation problem was solved by fitting a model by optimizing the sum of the, by the model, integrated intensity. This is generally an appeal- 
ing method to segment out regions of interest in SPECT images. It is fast, robust and of low complexity. Since there is little variation in the shape of the basal ganglia among individuals, we believe that a rigid model is sufficient. The shape is, however, interesting and in a future study the information shape can bring will be investigated more thoroughly. The initialization part in the optimization scheme is usually crucial. For most cases the proposed algorithm locates the global maximum. This is due to the fact that the region of the basal ganglia has higher intensity than the the rest of the brain. But for severe cases the maximal intensity distribution over the model can be in another part of the brain (normally the lower part) and therefore the initialization is important. The semi-automatic method uses the visual cortex to normalize the measurements. In the proposed method the median of the intensity outside the basal ganglia is used. If there are outliers, the median $m$ is proved to be more robust than the mean value [5] and motivates our choice of reference value.

\subsection{Quantification}

The features obtained by the proposed automatic method are similar to those obtained by the semi- automatic method. The most important features are the normalized mean intensity of left, right caudate and left, right putamen $\left(x_{6}-x_{9}\right.$ in Table 1). Other features of potential interest could include the standard deviation in each region, the minimum and the maximum. The reason for this is that, during the course of PD, a gradual loss of receptors first in the putamen, then the caudate nucleus is observed with considerable anterior-posterior gradient in these regions.

\subsection{Classification}

There are many different classifiers to choose among. Here Support Vector Machine (SVM) [6] was chosen because the underlying distribution is unknown. Another attractive property of a SVM is that it allows many features even if the training material contains few examples. Statistical methods usually require knowledge of the underlying distribution. Other, potentially better classifiers may be available. Different kernels were tested for the SVM. A polynomial kernel of degree 3, a radial based kernel with gamma equal to one, and a linear kernel all gave equally satisfactory results. For the implementations we have used the OSU-SVM toolbox for MATLAB [4]. The SVM gives for each sample a decision value. The samples are classified to one class or the other depending on whether this value is below or above a threshold. By varying this threshold a receiver operating characteristic (ROC) curve was obtained. The performance of the classification was measured as the area under the ROC curve. 


\section{$5 \quad$ Results and Conclusions}

Most parts of the program are implemented in MATLAB code and some in C. Using this non- optimised MATLAB code it takes less than one minute to extract the features and to do the classification (on a Pentium IV $1300 \mathrm{MHz}$ ). We have shown the feasibility of an automatic classification of abnormal images from patients likely to suffer from parkinsonism from SPECT data using the [123I]-FP-CIT isotope. To our knowledge this is the first completely automatic system that with a high probability can discriminate between abnormal from abnormal [123I]-FP- CIT SPECT-images. This pilot study of 89 cases also indicates that this fully automatic method performs equally well as the previous semi-automatic system. It has been demonstrated that a completely automated method can be used for the interpretation of [123I]-FP-CIT images.

\section{Acknowledgements}

This work has been financed by the SSF sponsored project 'Vision in Cognitive Systems' (VISCOS).

\section{References}

1. Jankovic, J., A.H., R., M.P., M.: Perl dp. the evolution of diagnosis in early parkinson disease. Parkinson Study Group. Arch Neurol 57 (2000) 369-372

2. Booij, J., Tissingh, G., Boer, G., Speelman, J., Stoof, J., Janssen, A.e.a.: 123-i-fpcit spect shows a pronounced decline of striatal dopamine transporter labelling in early and advanced parkinson's disease. Journal of Neurology, Neurosurgery, and Psychiatry 62 (1997) 133-140

3. Lokkegaard, A., Werdelin, L., Friberg, L.: Clinical impact of diagnostic spet investigations with a dopamine re-uptake ligand. Eur.J.Nucl.Med.Mol.Imaging 29 (2002) 1623-1629

4. Ma, J., Zhao, Y., Ahalt, S.: Osu svm classifier toolbox. (available at http://eewww.eng.ohio-state.edu/ $\sim$ maj/osu_svm)

5. Rice, J.: Mathematical Statistics and Data Analysis. second edn. Duxbury Press (1994)

6. Cristianini, N., Taylor, J.: An introduction to support vector machines. Cambridge (2000) 